\title{
Resonant Elements for Tunable Reflectarray Antenna Design
}

\author{
M. Y. Ismail and M. Inam \\ Wireless and Radio Science Centre (WARAS), Faculty of Electrical and Electronic Engineering, \\ Universiti Tun Hussein Onn Malaysia (UTHM), 86400 Batu Pahat, Johor, Malaysia \\ Correspondence should be addressed to M. Y. Ismail, yusofi@uthm.edu.my
}

Received 8 February 2012; Revised 5 April 2012; Accepted 5 June 2012

Academic Editor: Sandra Costanzo

Copyright ( 2012 M. Y. Ismail and M. Inam. This is an open access article distributed under the Creative Commons Attribution License, which permits unrestricted use, distribution, and reproduction in any medium, provided the original work is properly cited.

\begin{abstract}
This paper presents an accurate analysis of different configurations of reflectarray resonant elements that can be used for the design of passive and tunable reflectarrays. Reflection loss and bandwidth performances of these reflectarray elements have been analyzed in the X-band frequency range with the Finite Integral Method technique, and the results have been verified by the waveguide scattering parameter measurements. The results demonstrate a reduction in the phase errors offering an increased static linear phase range of $225^{\circ}$ which allows to improve the bandwidth performance of single layer reflectarray antenna. Moreover a maximum dynamic phase range of $320^{\circ}$ and a volume reduction of $22.15 \%$ have been demonstrated for a $10 \mathrm{GHz}$ reflectarray element based on the use of rectangular patch with an embedded circular slot.
\end{abstract}

\section{Introduction}

Due to the modern day need for long distance communications, higher data rate and finer radar imaging resolution, high gain or large aperture antennas are usually required. Deep space exploration and earth remote sensing are few missions of NASA where the antenna performance requirements are increasing in order to extract more information. On the other hand, lower mass and smaller stowage volume for the space craft antennas are demanded in order to reduce payload weight and required shroud space to minimize overall launch cost. Traditionally high gain applications have relied upon parabolic reflectors and phased arrays [1]. However, due to the curvature of their surface, parabolic reflectors are difficult to be manufactured in many cases at higher microwave frequencies [2]. The shape of the parabolic reflector also causes an increased weight and size of the antenna. Moreover, it has also been demonstrated in [3] that wide-angle electronic beam scanning cannot be achieved using a parabolic reflector. On the other hand, high gain phased array antennas offer the possibility to electronically scan the main beam along wide angle positions provided they are equipped with controllable phase shifters. However in order to reduce the problem of power inefficiency due to the problem of high loss beamformers and phase shifters, suitable amplifier modules have to be integrated with the array antennas. These amplifier modules are usually of high cost so making phased array antennas a very expensive solution for high gain applications. Therefore in order to eliminate these problems, a flat and low cost microstrip array antenna, known as reflectarray, has been acknowledged as a potential alternative to these traditionally used high gain antennas. Reflectarray in its basic form is a combination of a flat reflector and an array of microstrip patches illuminated by a primary feed horn. Reflectarray can achieve a wideangle electronic beam scanning. Direct Broadcast Satellites (DBS) and Multibeam Antennas (MBAs) are also considered as potential applications of reflectarrays. Moreover they can also act as amplifying arrays by including an amplifier in each reflectarray element [4].

Despite of the large number of reflectarrays advantages, the factors that limit its use in some applications are the limited bandwidth and high loss performance. Many different techniques have been proposed in order to increase the bandwidth performance of reflectarrays and up to $15 \%$ bandwidth has been reported [3]. Recently the authors have demonstrated the feasibility of designing a reflectarray with different types of slots embedded in a rectangular 


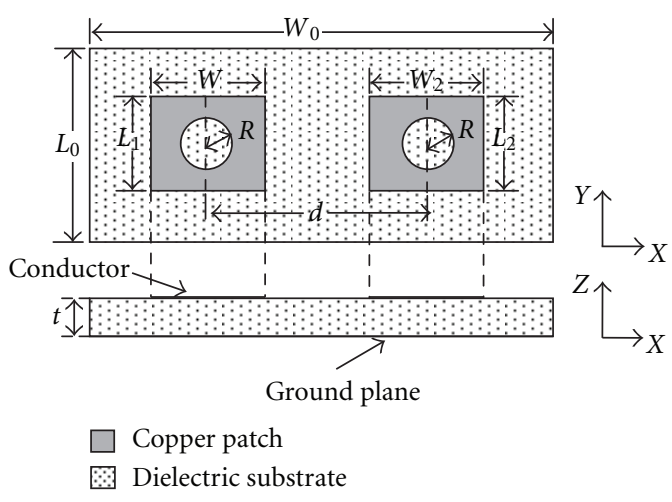

FIgURE 1: Geometry of a two patch element unit cell reflectarray ( $L_{1}=L_{2}$ is length of patch, $W_{1}=W_{2}$ is width of patch, $R$ is radius of circular slot, $L_{0}$ and $W_{0}$ are the length and width of substrate, resp., $t$ is thickness of substrate, and $d$ is the interelement spacing).

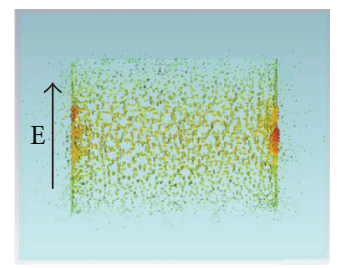

(a)

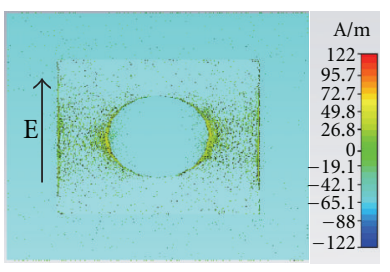

(b)
FIGURE 2: Surface current distribution on (a) rectangular patch of reflectarray and (b) reflectarray rectangular patch element with circular slot in the centre.

patch [5]. In this work, a reflectarray element based on the use of a rectangular patch with an embedded circular slot is proposed. The reflection losses and bandwidth performances of X-band elements are obtained through simulations and measurements. The proposed reflectarray design is also integrated with PIN diodes in order to obtain a tunable configuration.

\section{Proposed Reflectarray Configuration}

In order to improve the performance of reflectarray and to provide resonant elements for tunable reflectarray antenna design, a circular slot has been introduced in the centre of the reflectarray patch element. Figure 1 shows the top and the side view of the reflectarray unit cell composed by two patch elements. The circular slot elements have the same dimensions and are designed on a dielectric substrate with a thickness $(t)$. The interelement spacing $(d)$ between the two patch elements is kept to be half wavelength in order to minimize the mutual coupling effects.

Figure 2 shows the current distribution on the single reflectarray element obtained by using CST Microwave Studio (MWS). In this work a Rogers RT/duroid 5880 substrate with a thickness of $0.508 \mathrm{~mm}$ has been used to design an infinite reflectarray at $10 \mathrm{GHz}$. Figure 2 (a) shows that the current amplitude is maximum at the centre of the top surface of conducting rectangular patch element while

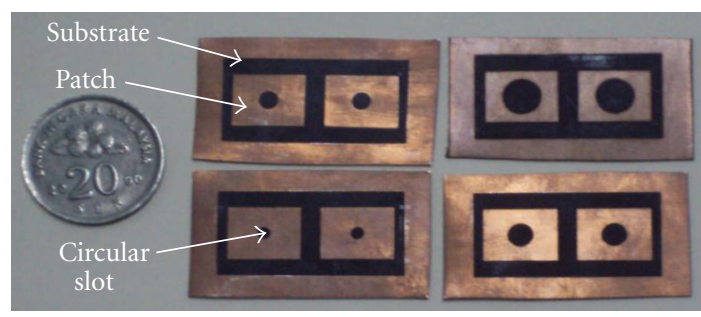

Figure 3: Fabricated unit cells rectangular patch elements embedded with different circular slots.

Figure 2(b) shows a clear modification of surface current distribution when a circular slot is embedded in the centre of patch element. This phenomenon of surface current modification is exploited in this work to tune the reflection phase of the proposed reflectarray element.

Measurements of the scattering parameters for infinite reflectarrays fabricated using Rogers RT/duroid 5880 have recently been demonstrated in [6] for operation in X-band frequency range. Waveguide simulator technique has been used to represent a two-patch element unit cell with an infinite reflectarray. For this work unit cells reflectarrays have been fabricated by integrating the circular slots of different radius with rectangular patch elements as shown in Figure 3. The dimensions of each radiating element, depicted in Figure 2, are fixed to the following values: $W_{1}=W_{2}=$ $10 \mathrm{~mm}, L_{1}=L_{2}=9.4 \mathrm{~mm}$. In order to tune the phase of the field reflected by the elements, the radius of each circular slot is varied from $1 \mathrm{~mm}$ to $3 \mathrm{~mm}$. Reflection losses and phase range performances of the designed unitary cell are discussed in the following sections. Furthermore, in the last part of this work, the designed elements are integrated with a PIN diode in order to demonstrate the effectiveness of the proposed configuration to design tunable reflectarray antennas.

2.1. Reflection Loss Performance. Figure 4 depicts the measured and simulated reflection loss for different radius of circular slots and reflection loss for a patch without slot. A significant change in the resonant frequency from $10 \mathrm{GHz}$ to 8.5 GHz has been observed as the element was varied from the case without slot to the one having a slot radius equal to $3.0 \mathrm{~mm}$. It has been shown that an increase in the slot radius from $1.0 \mathrm{~mm}$ to $3.0 \mathrm{~mm}$ also caused an increase in the measured reflection loss from $1.7 \mathrm{~dB}$ to $2.65 \mathrm{~dB}$.

Moreover the trend of the loss performance of both measured and simulated reflectarrays is in good agreement. The change in resonant frequency and reflection loss with variable radius of slots is due to the change in electrical dimensions which in turn modifies the surface current distribution on the patch element of a reflectarray. It can also be observed that the measured reflection loss is higher than the simulated reflection loss because of the losses introduced by the interconnections and material properties.

2.2. Phase Range Performance. In order to compare the phase range performance of different reflection phase curves, a 
TABle 1: Performance comparison for different resonant elements of reflectarray.

\begin{tabular}{|c|c|c|c|c|c|}
\hline \multirow{2}{*}{ Resonant element } & \multirow[b]{2}{*}{$\begin{array}{l}\text { Reflection } \\
\text { loss }(\mathrm{dB})\end{array}$} & \multicolumn{3}{|c|}{ Dynamic phase range } & \multirow{2}{*}{$\begin{array}{l}\text { Volume reduction } \\
\text { at } 10 \mathrm{GHz}(\%)\end{array}$} \\
\hline & & $f_{o}(\mathrm{GHz})$ & $\begin{array}{c}\text { Measured } \\
\text { (degrees) }\end{array}$ & $\begin{array}{c}\text { Simulated } \\
\text { (degrees) }\end{array}$ & \\
\hline Rectangular patch & 1.50 & - & - & - & - \\
\hline Circular slot $(R=1.5 \mathrm{~mm})$ & 1.75 & 9.75 & 104 & 110 & 5.24 \\
\hline Circular slot $(R=2.0 \mathrm{~mm})$ & 2.20 & 9.45 & 290 & 298 & 9.19 \\
\hline Circular slot $(R=2.5 \mathrm{~mm})$ & 2.50 & 9.25 & 310 & 314 & 15.76 \\
\hline Circular slot $(R=3.0 \mathrm{~mm})$ & 2.65 & 9.10 & 320 & 323 & 22.15 \\
\hline
\end{tabular}

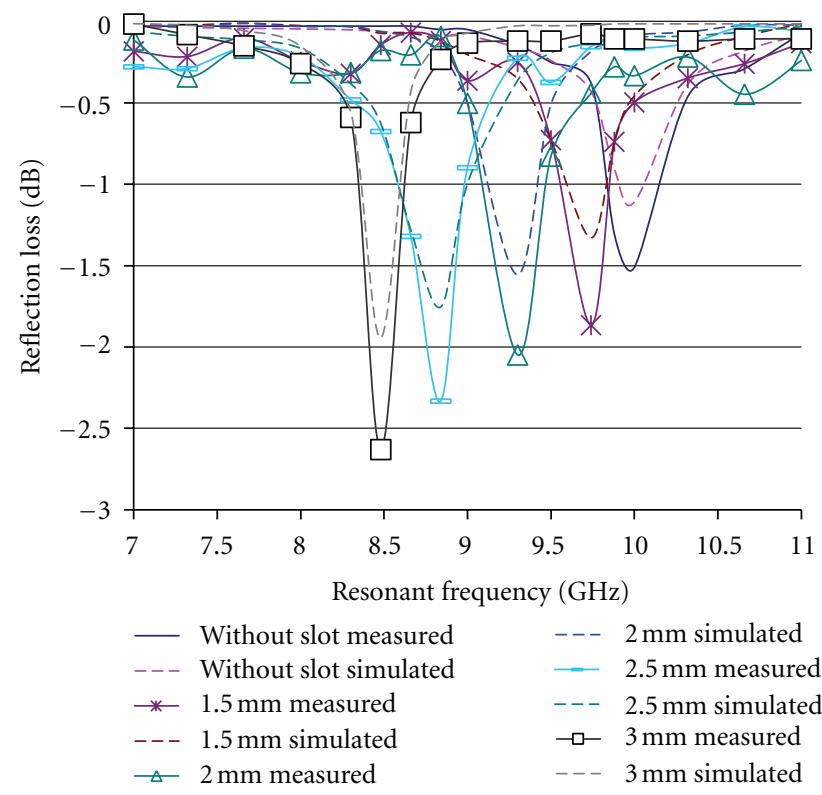

Figure 4: Measured and simulated reflection loss for patch without slot and patch with different radius of circular slots in the centre of patch.

figure of merit (FoM) has been defined which is related to the slope of the phase curves. The FoM is given by

$$
\mathrm{FoM}=\frac{\Delta \phi}{\Delta f},
$$

where $\Delta \phi$ is the change in the reflection phase in degrees and $\Delta f$ is the change in the reflectarray antenna resonant frequency in $\mathrm{MHz}$, thus FoM is calculated here in ${ }^{\circ} / \mathrm{MHz}$. For a reflectarray antenna the FoM or the slope of the phase curve around the resonance is required to be minimum in order to have better bandwidth performance. Measured and simulated reflection phases for different radius of circular slots $(R)$ embedded in the centre of patch and for a patch without slot are shown in Figure 5. It can be observed from Figure 5 that as the unit cell was varied from patch element without slot to a circular slot of $3.0 \mathrm{~mm}$ radius, the slope of the reflection phase curve increases from $0.24^{\circ} / \mathrm{MHz}$ to

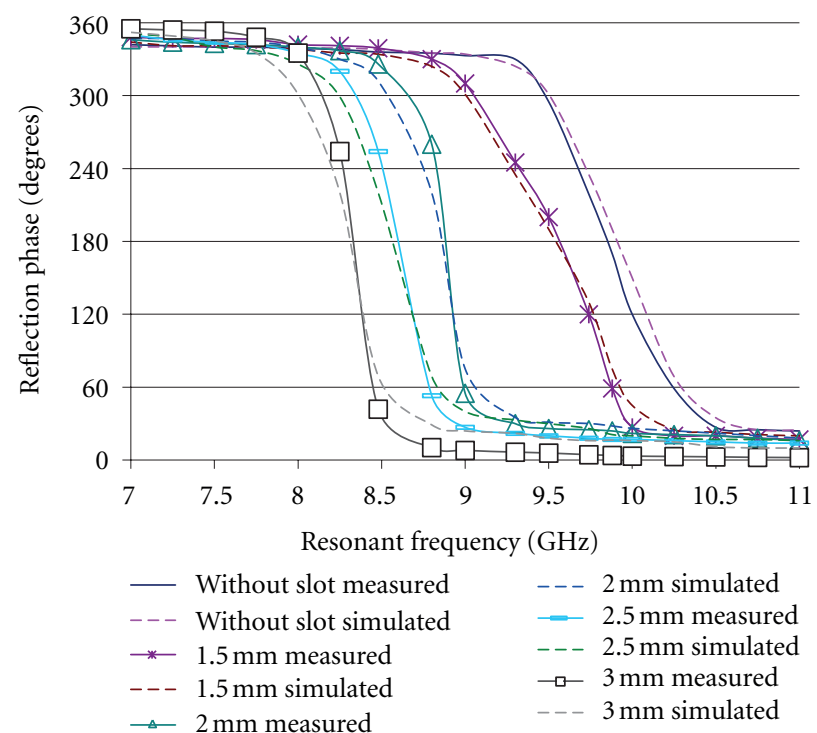

FIGURE 5: Measured and simulated reflection phase for patch without slot and patch with different radius of circular slots in the centre of patch.

$1.04^{\circ} / \mathrm{MHz}$. This is due to the fact that the surface current distribution is modified by the introduction of the slot in the patch. In fact, the surface current density and the electric field intensity decrease by increasing the circular slot radius.

A further parameter adopted to evaluate the phase curves behavior is the dynamic phase range. This parameter can be defined as the difference between two different phase curves, respectively, computed for the patch without slot and the patch with a variable radius slot. The phase difference is evaluated at the mean resonant frequency (Figure 6(a)). Table 1 shows measured and simulated dynamic phase range for different radius of circular slots. It can be observed from Table 1 that a maximum measured dynamic phase range of $320^{\circ}$ is shown by an element with a circular slot of $3.0 \mathrm{~mm}$ radius. Another parameter considered for the comparison of patch with slot and patch without slot is the volume reduction which provides the feasibility of miniaturizing the reflectarray antenna. It is measured as the percentage difference between the volume of the proposed 


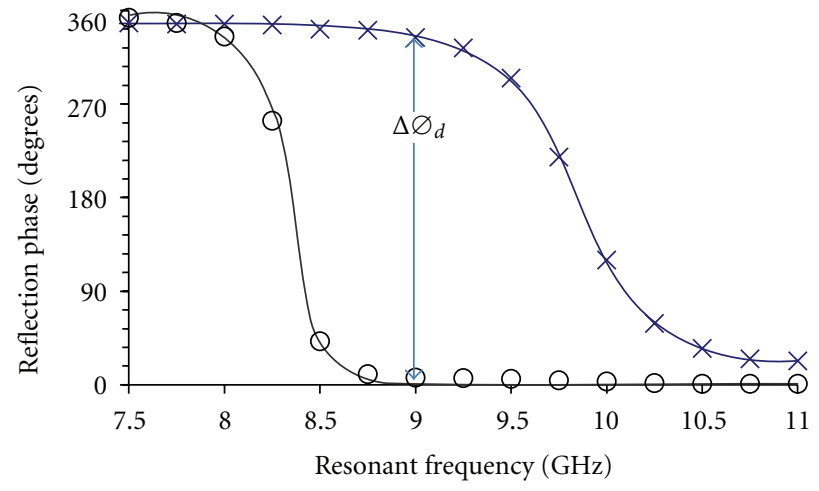

(a)

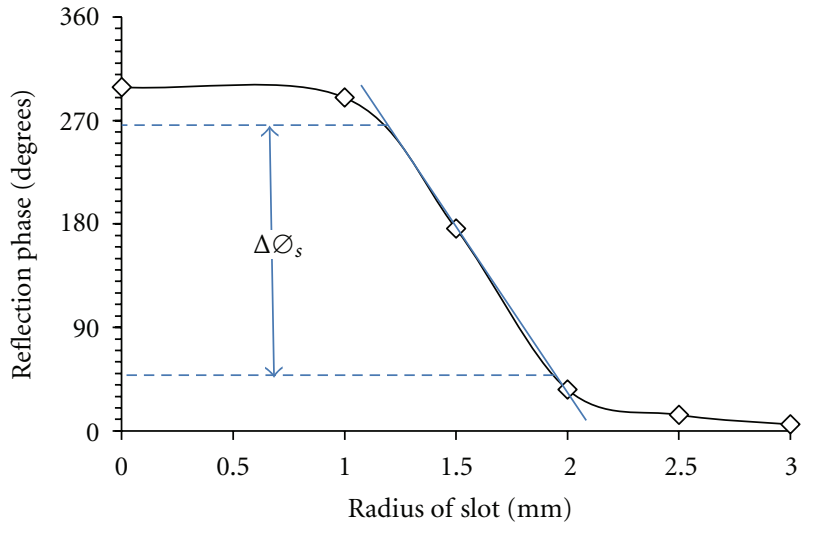

(b)

FIGURE 6: (a) Dynamic phase range, (b) measured static linear phase range of phase shift versus slot size curve for circular slot configurations at $9.75 \mathrm{GHz}$.

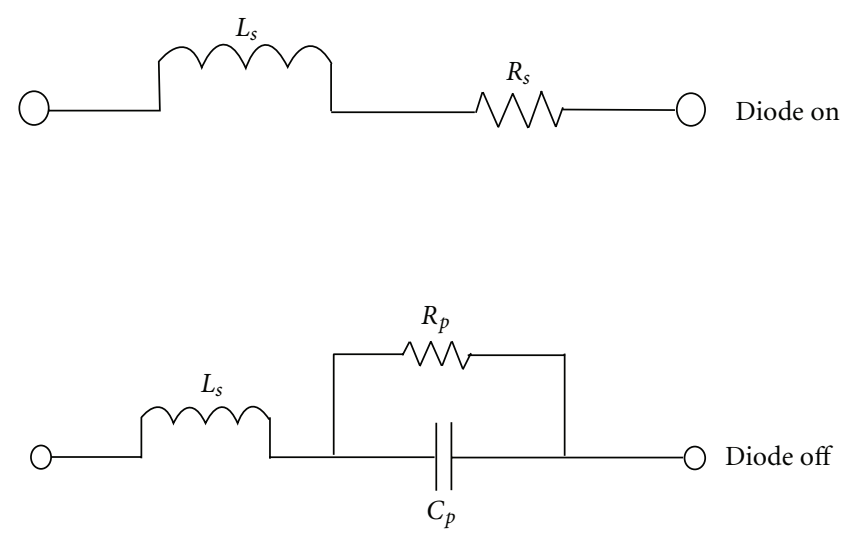

(a)

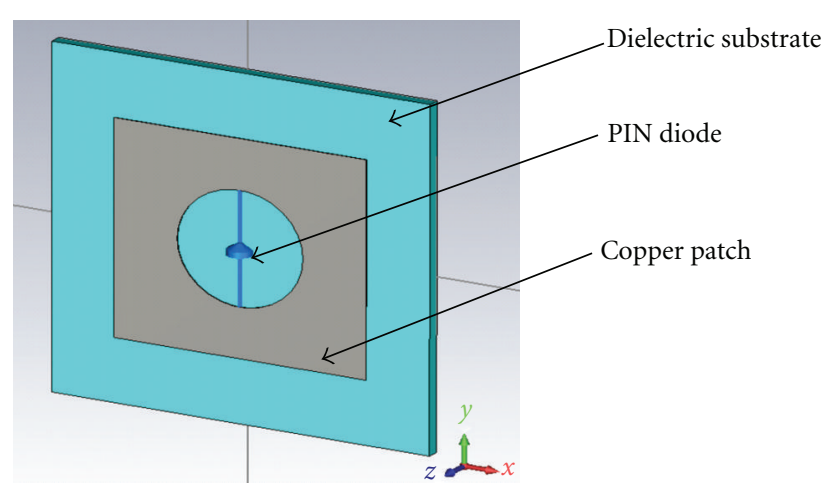

(b)

FIgURE 7: (a) On and off states of a PIN diode, (b) proposed design with integrated PIN diode.

slot embedded patch element and the rectangular patch element designed at $10 \mathrm{GHz}$. A maximum volume reduction of $22.15 \%$ is shown to be achieved by an element with a circular slot of $3.0 \mathrm{~mm}$.

In order to compare the results of the reflection phase plots produced in this work, the static linear phase range $\left(\Delta \emptyset_{s}\right)$ defined in Figure 6(b) has been used. The previous studies $[6,7]$ which proposed the slots in the ground plane demonstrated a simulated static linear phase range of $180^{\circ}$ and $210^{\circ}$, respectively, for single layer structures. The results depicted in Figure 6(b) demonstrate that an increased measured static linear phase range of $225^{\circ}$ is achieved using slots of variable radius in the patch element. Therefore due to the increased attainable static linear phase range, the phase error contribution in the reduction of reflectarray bandwidth can be minimized by using the proposed slotted configurations.

\section{Design of a Tunable Reflectarray Antenna Using PIN Diodes}

The reflectarray configuration proposed in Section 2 can be loaded with diodes in order to design tunable planar reflectarrays for beam steering applications. A potential type of diode that can be used for frequency tunability is PIN diode. The on and off states of a PIN diode, result in different equivalent lumped circuits as shown in Figure 7(a). In the case of the on state the pin diode provides a series resistance which affects the reflection loss of the reflectarray and therefore needs to be kept low. On the other hand, in the off state the PIN diode provides a low capacitance which affects the tunability performance of the reflectarray element. In order to verify the functionality of the electronically switchable reflectarray design, PIN diodes have been integrated with circular slots as shown in Figure 7(b). 


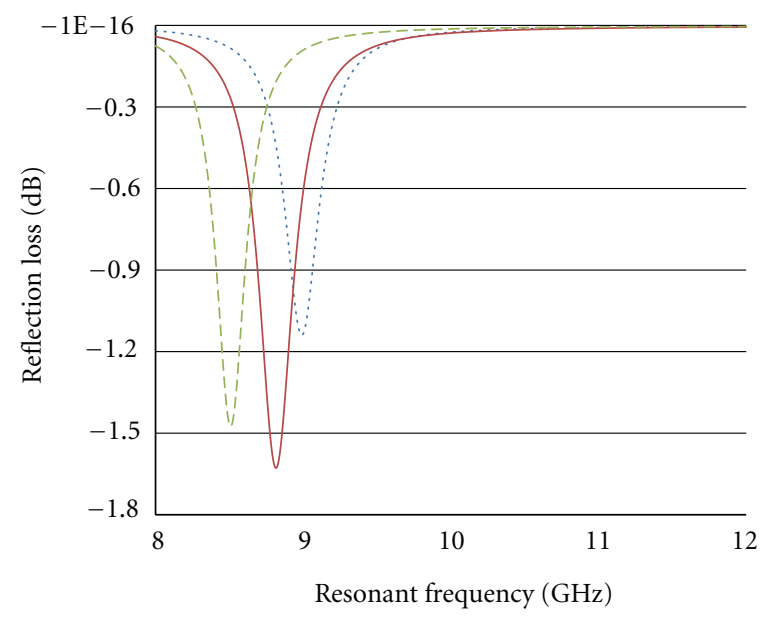

(a)

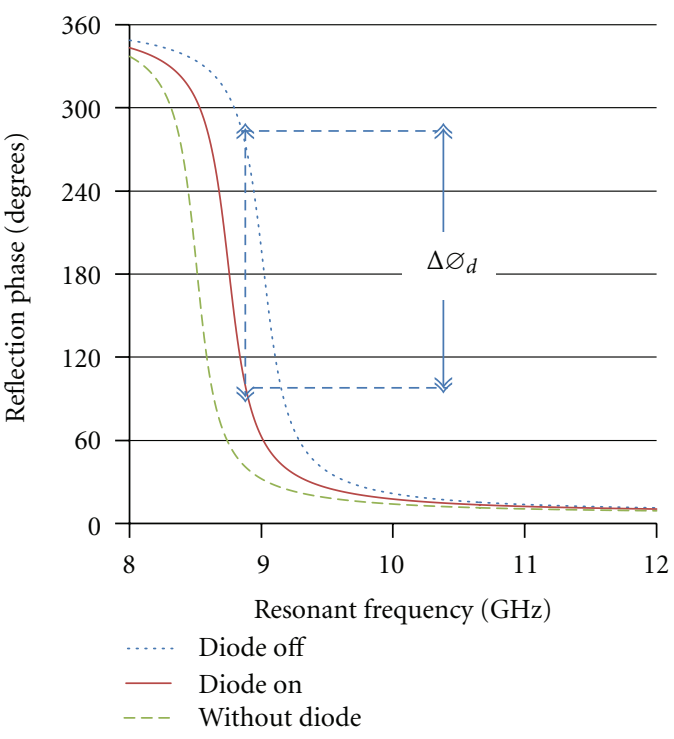

(b)

FIGURE 8: Reflection loss and reflection phase curves for tunable reflectarray design with PIN diodes.

In this work, a circular slot with $R=2.5 \mathrm{~mm}$ has been integrated with an APD 0805-000 PIN diode. The APD 0805-000 PIN diode can operate at high frequencies and has low capacitance and low series resistance which provides lower losses and a higher degree of tunability. Figure 8 shows reflection loss and refection phase curves for the design without diode and with two different states of a PIN diode. It can be observed from Figure 8 that the on state of PIN diode provides a higher reflection loss equal to $1.64 \mathrm{~dB}$ with respect to the other states. A frequency tunability of $270 \mathrm{MHz}$ and a dynamic phase range of $171^{\circ}$ at $8.88 \mathrm{GHz}$ can be observed by the results shown in Figure 8 . The results demonstrate the feasibility of effectively utilizing the proposed slot configurations with PIN diodes for the design of tunable reflectarray antennas.

\section{Conclusion}

A reflectarray element based on the use of a rectangular patch with an embedded circular slot has been proposed. It has been demonstrated that the configuration can be effectively utilized for the design of passive and tunable reflectarrays with low loss and high bandwidth performance. From the measurements of the slot embedded patch element, it can be concluded that proposed configuration can be employed for the miniaturization of the reflectarrays as a wide range of resonant frequencies can be achieved without varying the size of the patch element. Moreover the reduction in the phase errors produced by the limited phase range and the possibility of designing a miniaturized reflectarray has also been demonstrated using the proposed configurations. Preliminary investigations for the integration of slots and PIN diodes have shown that the dynamic phase range can be utilized to design electronically controllable reflectarrays with progressive phase distribution.

\section{Acknowledgments}

This research work is fully funded by Prototype Research Grant Scheme (PRGS VOT 0904) and Best Awarded Fundamental Research Grant Scheme (FRGS VOT 0983), Ministry of Higher Education, Malaysia. The authors would like to thank the staff of Wireless and Radio Science Centre (WARAS), Universiti Tun Hussein Onn Malaysia (UTHM) for the technical support.

\section{References}

[1] G. D. G. Berry, R. G. Malech, and W. A. Kennedy, "The reflect array antenna," IEEE Transactions on Antennas and Propagation, vol. 11, pp. 645-651, 1963.

[2] J. Huang and J. A. Encinar, Reflectarray Antennas, Wiley Interscience, 2007.

[3] J. A. Encinar, M. Arrebola, M. Dejus, and C. Jouve, "Design of A 1-metre reflectarray for DBS application with $15 \%$ bandwidth," in Proceedings of the European Conference on Antennas and Propagation (EuCAP '06), pp. 1-5, Nice, France, November 2006.

[4] M. E. Bialkowski, A. W. Robinson, and H. J. Song, "Design, development, and testing of X-band amplifying reflectarrays," IEEE Transactions on Antennas and Propagation, vol. 50, no. 8, pp. 1065-1076, 2002.

[5] M. Y. Ismail, M. Inam, and J. Abdullah, "Design optimization of reconfigurable reflectarray antenna based on phase agility technique," in Proceedings of the 3rd Conference on Antenna \& RCS Measurement (ATMS '10), vol. 33, pp. 01-05, Delhi, India, 2010. 
[6] M. I. Abbasi and M. Y. Ismail, "Reflection loss and bandwidth performance of X-band infinite reflectarrays: simulations and measurements," Microwave and Optical Technology Letters, vol. 53, no. 1, pp. 77-80, 2011.

[7] H. Rajagopalan, Y. Rahmat-Samii, and W. A. Imbriale, "RF MEMS actuated reconfigurable reflectarray patch-slot element," IEEE Transactions on Antennas and Propagation, vol. 56, no. 12, pp. 3689-3699, 2008. 

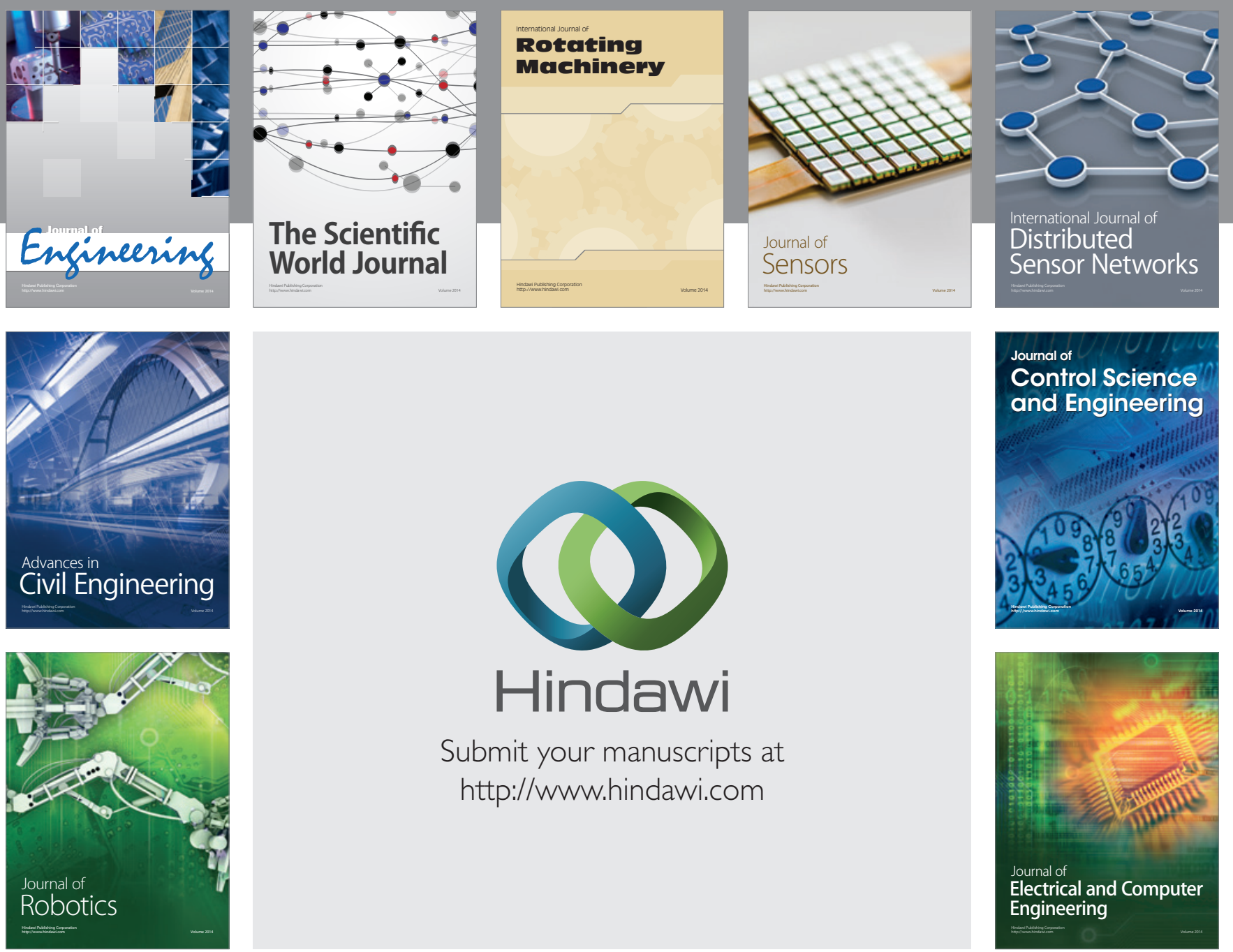

Submit your manuscripts at

http://www.hindawi.com
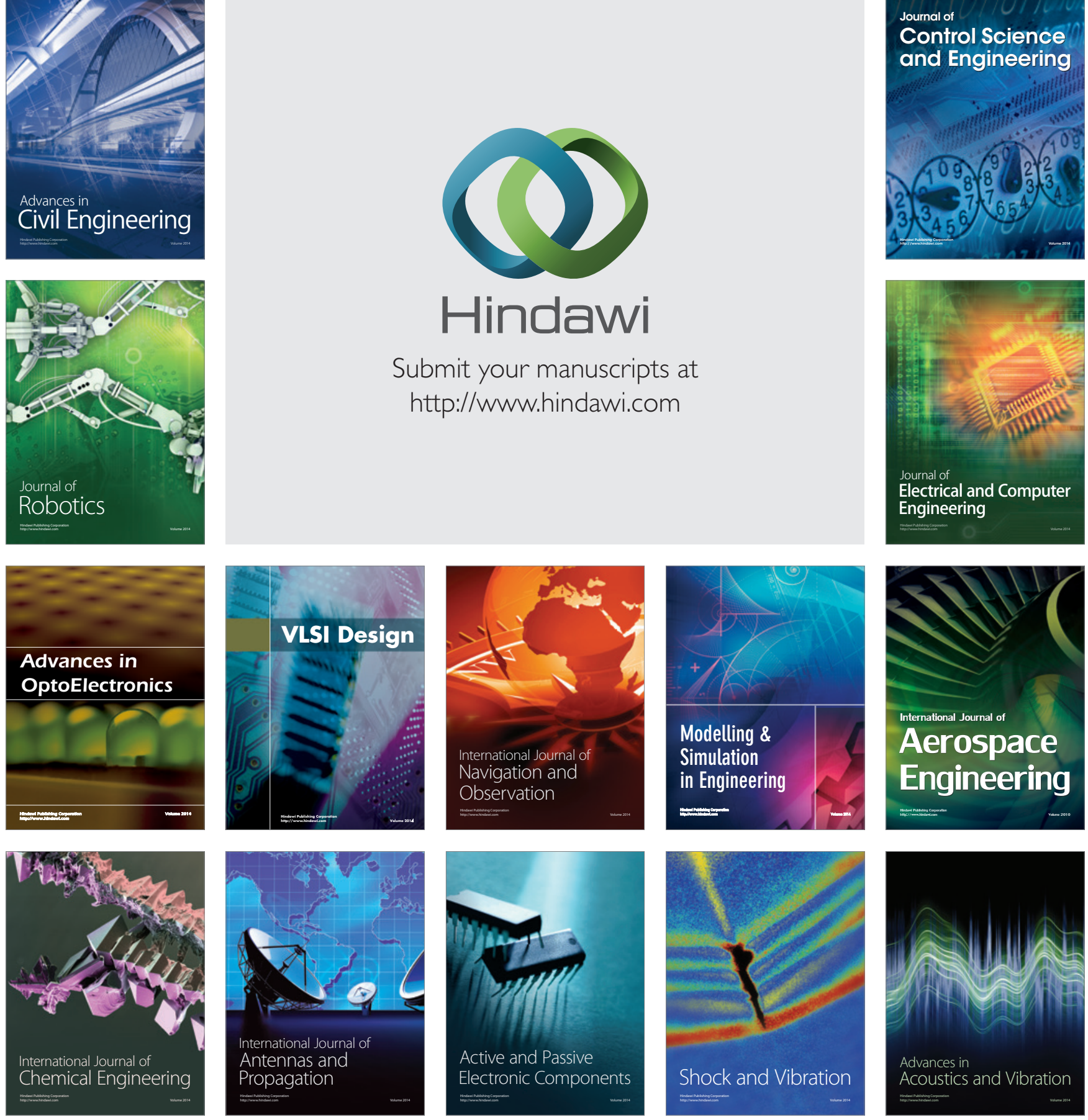